Journal of

\section{Synchrotron}

Radiation

ISSN 0909-0495

Received 28 October 2009

Accepted 12 February 2010

\title{
X-ray magnetic circular dichroism measurements using an X-ray phase retarder on the BM25 A-SpLine beamline at the ESRF
}

\author{
Roberto Boada, ${ }^{\mathrm{a}, \mathrm{b} *}$ María Ángeles Laguna-Marco, ${ }^{\mathrm{c}}$ Jon Ander Gallastegui, ${ }^{\mathrm{d}, \mathrm{e}}$ \\ Germán R. Castro $^{d, e}$ and Jesús Chaboy ${ }^{a, b}$ \\ a Instituto de Ciencia de Materiales de Aragón, Consejo Superior de Investigaciones Científicas, \\ CSIC-Universidad de Zaragoza, 50009 Zaragoza, Spain, ${ }^{\mathbf{b}}$ Departamento de Física de la Materia \\ Condensada, Universidad de Zaragoza, 50009 Zaragoza, Spain, 'Advanced Photon Source, \\ Argonne National Laboratory, Argonne, IL 60439, USA, 'd Instituto de Ciencia de Materiales de \\ Madrid, Consejo Superior de Investigaciones Científicas, Cantoblanco, 28049 Madrid, Spain, and \\ ${ }^{\mathbf{e}}$ SpLine, Spanish CRG Beamline at the European Synchrotron Radiation Facility, BP 220, F-38043 \\ Grenoble Cedex, France. E-mail: rboada@unizar.es
}

Circularly polarized X-rays produced by a diamond X-ray phase retarder of thickness $0.5 \mathrm{~mm}$ in the Laue transmission configuration have been used for recording X-ray magnetic circular dichroism (XMCD) on the bending-magnet beamline BM25A (SpLine) at the ESRF. Field reversal and helicity reversal techniques have been used to carry out the measurements. The performance of the experimental set-up has been demonstrated by recording XMCD in the energy range from 7 to $11 \mathrm{keV}$.

Keywords: X-ray magnetic circular dichroism; X-ray phase retarder; polarization tunability; magnetic polarization.

\section{Introduction}

X-ray magnetic circular dichroism (XMCD), the difference in absorption for left- and right-circularly polarized X-rays by a magnetized sample, constitutes nowadays an outstanding tool for the study of magnetism. In bending-magnet sources at synchrotron radiation facilities a high rate of circularly polarized X-rays, essential to XMCD, can be obtained before monochromatization (Ishikawa, 1988) by selecting the radiation emitted above or below the plane of the electron storage ring (Schütz et al., 1987). The degree of circular polarization increases with vertical angular aperture but unfortunately at the expense of flux (Duke, 2000; Stöhr et al., 1998).

The flux and tunability of polarized X-rays have been improved by using specific insertion devices (IDs) such as elliptical multipole wigglers (Kawata et al., 1988; Yamamoto et al., 1989) or helical undulators (Goulon et al., 1995). However, quick reversal of the photon helicity at these exotic IDs is difficult because this procedure usually involves the rearrangement of magnet arrays.

The development of diffractive X-ray phase retarders (XPRs) (Hirano et al., 1991, 1994; Giles et al., 1994) using transmission geometry has improved the switching ratio of $\mathrm{X}$-ray polarization states. It has also opened the possibility of extending the spin-dependent spectroscopies to every beamline by combining XPR and linearly polarized radiation emitted by undulators (Maruyama, 2001; Suzuki et al., 1998; Lang \& Srajer, 1995; Haskel et al., 2001; Pizzini et al., 1998) or bending magnets (Bouchenoire et al., 2003). Moreover, X-ray phase plates can provide well defined polarization without being affected by the finite emittance of the electron beam circulating in the storage ring (Hirano et al., 1991). Another improvement of XPR usage is given by the helicity modulation technique which combines a fast helicity reversal switching with a lock-in detection, optimizing the signal-to-noise ratio (Hirano et al., 1992; Suzuki et al., 1998, 2003).

In this work we report on the first XMCD measurements performed on the BM25-SpLine beamline at the ESRF. This is a non-XMCD dedicated beamline and the set-up was performed along the duration of an almost standard beam time (ten days) with the handicap that it is a bending-magnet beamline with a large angular divergence. Linearly polarized $\mathrm{X}$-rays were converted into circularly polarized X-rays by using a $0.5 \mathrm{~mm}$-thick diamond X-ray phase retarder working in Laue geometry. Both magnetic field reversal and helicity reversal modes have been used covering an energy range from 7 to $11 \mathrm{keV}$. The performance of the designed set-up is illustrated by showing XMCD spectra recorded at the Gd and Ho $L_{2}$-edges and at the Ge $K$-edge, a nominally non-magnetic atom, in the case of $R\left(\mathrm{Fe}_{1-x} \mathrm{Ge}_{x}\right)_{2}$ (where $R$ is a rare-earth atom) Laves phase compounds.

\section{X-ray transmission phase plates}

The principle of X-ray transmission phase plates is fully described by the dynamical theory of X-ray diffraction that 


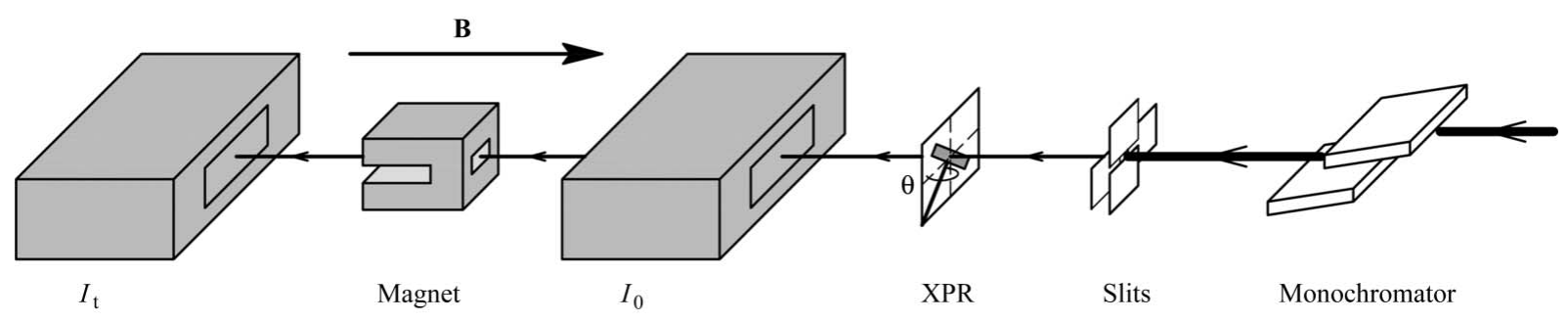

Figure 1

Schematic layout of the set-up for XMCD measurements. This figure is in colour in the electronic version of this paper.

allows for the full interference of electromagnetic waves in a periodic crystal (Batterman \& Cole, 1964). It is known that perfect crystals close to the Bragg condition are birefringent, i.e. the $\pi$ - and $\sigma$-polarization components of the electric vector of the incident beam have different phase velocities when they propagate through the crystal that introduces a phase shift $\delta$ between them. If the diffraction plane is inclined by an angle $\varphi$ with respect to the electric field of the incident linearly polarized X-ray beam, the circular polarization rate $P_{\mathrm{C}}$ depends on the phase shift $\delta$ in the form $P_{\mathrm{C}}=\sin (\delta) \sin (2 \varphi)$.

To achieve full circularly polarized X-rays both $\pi$ and $\sigma$ components must be coherently excited with equal electric field amplitude $(\varphi=\pi / 4)$ and the phase shift between them must be $\pi / 2$. The latter condition can be fulfilled for each energy by moving out of the Bragg condition by a proper offset angle $\Delta \theta$ since for a chosen phase plate thickness and diffraction configuration this offset at the circular polarization condition depends on energy as $\sim E^{-4}$ (see Hirano et al., 1992).

\section{Experimental XMCD set-up}

The beamline BM25 (SpLine) (Castro, 1998) at the ESRF is a bending-magnet beamline split into two different branches each with a horizontal opening angle of $2 \mathrm{mrad}$, one (branch A) centered at $3.5 \mathrm{mrad}$ and with a critical energy of $9.6 \mathrm{keV}$, and the other (branch B) centered at $10.5 \mathrm{mrad}$ with a critical energy of $20.6 \mathrm{keV}$. Branch $\mathrm{A}$ is dedicated to X-ray absorption spectroscopy and high-resolution X-ray powder diffraction. A schematic view of the beamline at the XMCD set-up is given in Fig. 1. The double-crystal monochromator (DCM) used for this branch is a pseudo channel-cut type with two fixed $\mathrm{Si}$ (111) crystals moved together by a simple goniometer circle in the $(-n,+n)$ configuration. The first monochromator crystal is water cooled while the second is kept at room temperature. The second crystal can be finely tilted with respect to the first one in three perpendicular axes. The pitch angle (concentric to the Bragg angle of the crystal) can be regulated during an energy scan in order to keep the transmission of the monochromator optimized during the whole scan, and to reduce the higher-order harmonic content of the beam if necessary. To guarantee a stable beam position and shape on the sample during the energy scan, pre-sample slits (typically of size $1 \mathrm{~mm} \times 1 \mathrm{~mm}$ ) are placed in front of the

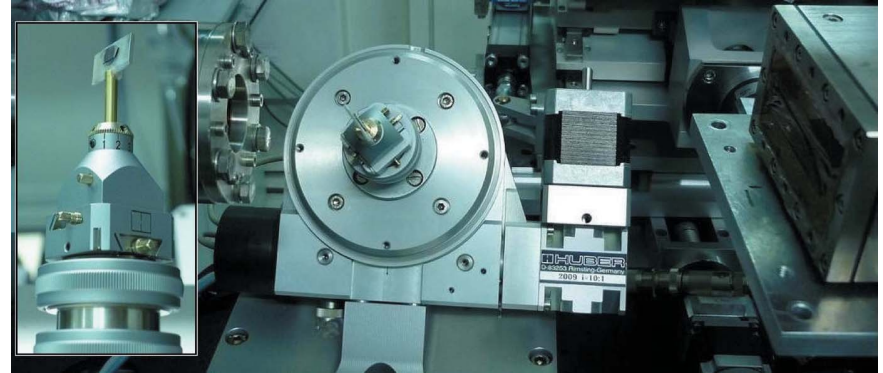

Figure 2

XPR holder and motor stage tilted $45^{\circ}$ away from the horizontal plane. This figure is in colour in the electronic version of this paper.

sample, and the focusing is tuned to keep the beam just larger than the slits in their position. In this way, even if the beam is moving slightly during the scan, the beam position and shape on the sample are fixed.

For the XMCD measurements a synthetic 111-diamond plate (Sumitomo Corporation) of thickness $0.5 \mathrm{~mm}$ was fixed using beeswax onto a crystal holder plate mounted on a standard pin goniometer (see Fig. 2). The rotation axis was tilted $45^{\circ}$ away from the polarization plane of the incident $\mathrm{X}$-ray beam. We have used the symmetric Laue geometry in which the $(2 \overline{2} 0)$ diffraction plane, perpendicular to the crystal surface, is chosen. Two translational motors ( $X$ and $Z$ axes) are used to center the diamond in the beam and a third one rotates the XPR to tune the Bragg condition ( $\theta$ rotation). In order to obtain circularly polarized X-rays the Bragg peaks are found for each energy point and then the XPR is tuned out of the diffraction condition by adding (subtracting) an offset angle $\Delta \theta$ to obtain left-circularly polarized (right-circularly polarized) light. ${ }^{1}$ A scintillation detector was used to detect the diamond (22) 0 ) Bragg peaks. The position of this detector was kept fixed for every energy range since its solid acceptance angle is large enough to collect the diffracted beam along a whole energy scan.

In-house-design magnet for transmission-mode measurements were made using $\mathrm{Nd}-\mathrm{Fe}-\mathrm{B}$ permanent magnets (see Fig. 3). The magnetic field could be varied by changing the gap between pole pieces. For these experiments the minimum gap,

\footnotetext{
${ }^{\mathbf{1}}$ We consider the atomic convention for the left- and right-handed sense of circularly polarized light (viewed from the source).
} 


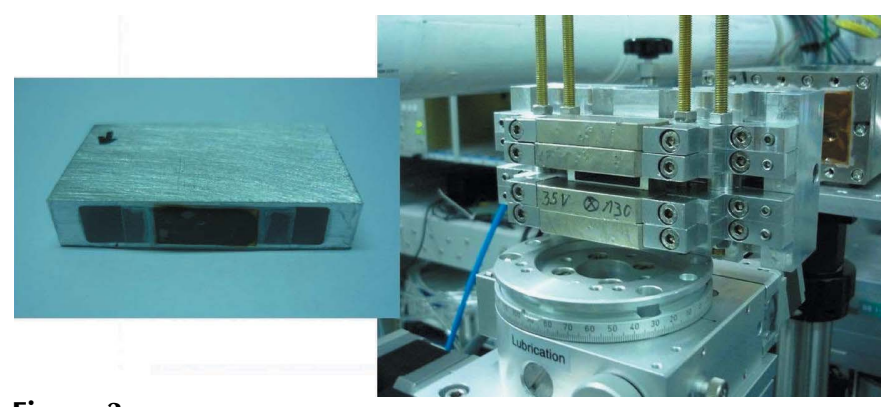

Figure 3

Sample holder and permanent magnet used. This figure is in colour in the electronic version of this paper.

$5 \mathrm{~mm}$, was chosen to obtain a $4.8 \mathrm{kOe}$ magnetic field at the sample position.

\section{XMCD measurements}

XMCD was measured on reference $\mathrm{GdFe}_{2}$ and $\mathrm{HoFe}_{2}$ samples at the rare-earth $L_{2}$-edges. These samples were chosen because their XMCD signals are well characterized and their magnitude and shape allow us to verify that the observed signals are not affected by any spurious signal or derivative
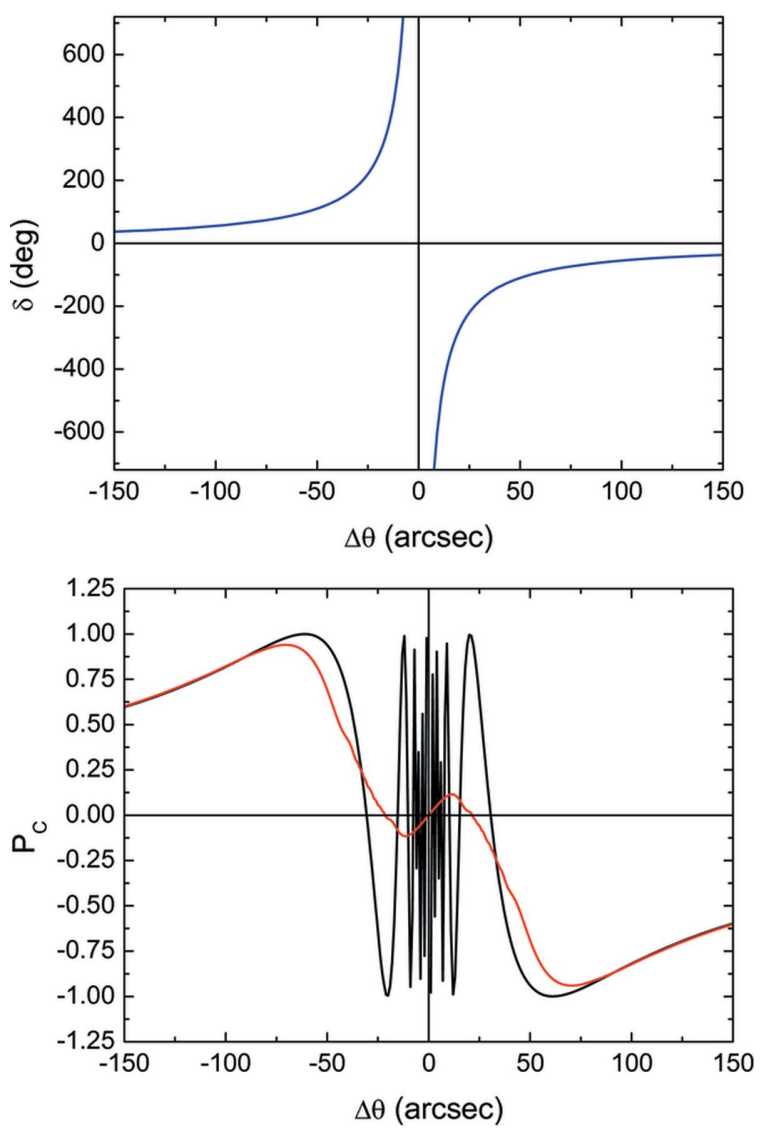

Figure 4

Phase shift (top) and circular polarization rate (bottom) curves as a function of the offset angle. The theoretical curve for $P_{\mathrm{C}}$ (black line) has been convoluted with a 50 arcsec FWHM Gaussian (red line) to consider the effective divergence of the beam. This figure is in colour in the electronic version of this paper. effect. Once the performance of the XMCD set-up was verified, the Ge $K$-edge XMCD was recorded on a $\mathrm{Gd}\left(\mathrm{Fe}_{0.9} \mathrm{Ge}_{0.1}\right)_{2}$ sample in which no magnetic role is a priori assigned to the Ge atoms.

The offset needed to obtain circular polarization conditions was chosen according to the theoretical curves for the circular polarization rate $P_{\mathrm{C}}=\sin \delta$. The theoretical $P_{\mathrm{C}}$ has been convoluted with a Gaussian function in order to account for the effective divergence of the incident beam, which causes a smearing of the polarization states through the spread of the $\delta$ phase shift. However, this effect is minimized by operating the phase plate at large offsets because $\delta$ is a slowly varying function of $\Delta \theta$ (see Fig. 4). The vertical divergence of the beamline has been estimated at about 10 arcsec, while the horizontal divergence has been estimated between 20 and 80 arcsec, depending on the size of the pre-sample slits and the focalization point. The importance of this parameter in selecting a stable offset angle is illustrated in Fig. 5 where the energy dependence of both the circular polarization rate and the offset angle for different FWHMs of the Gaussian used in the convolution (20, 50 and 80 arcsec) are shown.

For the experiments, XPR tuning was carried out as follows. At each absorption edge energy several Bragg peaks were recorded along the energy range of interest using a scintilla-
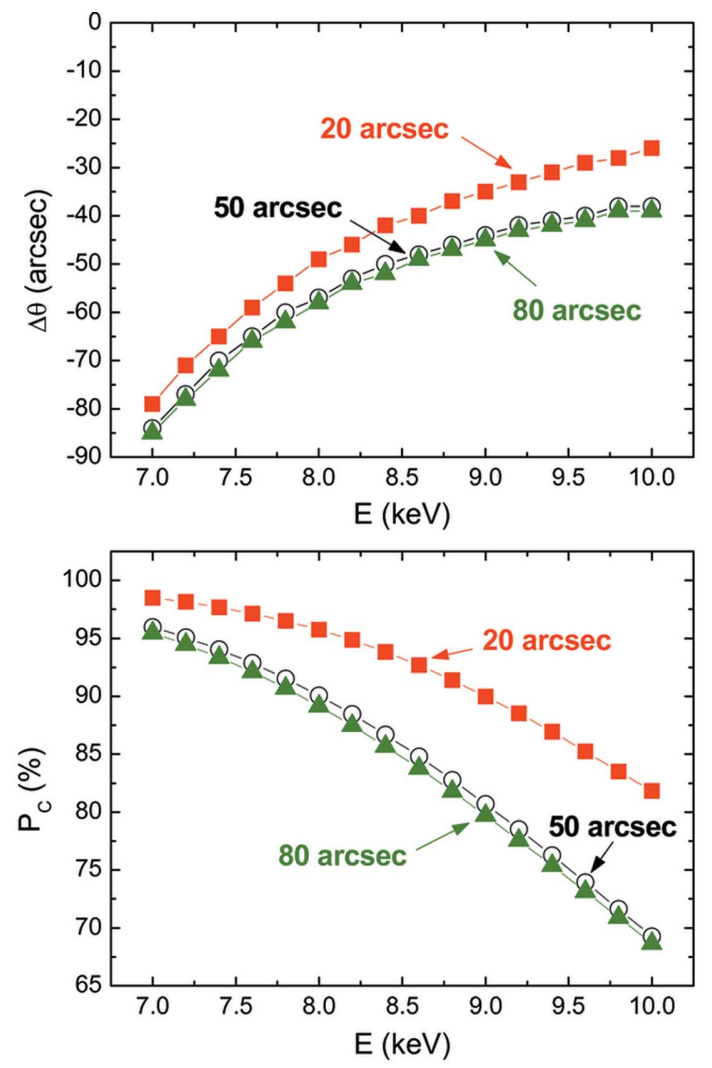

Figure 5

Dependence of the offset angle (top) and circular polarization rate (bottom) on the energy for different FWHM Gaussians used for the convolution: 20 (red solid squares), 50 (black open circles) and 80 arcsec (green solid triangles). This figure is in colour in the electronic version of this paper. 
tion detector. The angle required to move the XPR during each energy scan was obtained by fitting the peak position versus energy to an $\arcsin (A / E)$ function, where $A$ is a constant. Nevertheless, we have verified that for a small energy window $(\sim 100 \mathrm{eV})$, as we use to record the XMCD spectra, the error introduced by a linear approximation is negligible. Moreover, we have considered a fixed offset for the whole energy scan. In the case of a $200 \mathrm{eV} \mathrm{XMCD} \mathrm{scan} \mathrm{at} \mathrm{the}$ Ho $L_{2}$-edge the offset angle varies by $\sim 2$ arcsec. For these reasons the angle needed to move the diamond phase plate during the energy scanning has been varied linearly.

It should be noted that up to now we have considered (see Fig. 5) the offset $\Delta \theta$ as the small angle out of the Bragg condition that provides the maximum value of the circular polarization rate, i.e. the absolute maximum (minimum) of the $P_{\mathrm{C}}$ curve to obtain right-circularly polarized (left-circularly polarized) light (see bottom panel of Fig. 4). Actually, this position is not the best choice since it is not stable and small variations could cause undesirable fluctuations in the polarization rate. For this reason it is better to consider a larger value for the offset at the expense of circular polarization rate, to avoid the region close to the diffraction condition. Finally, we have also tested the transmissivity of the XPR for different energy ranges. The results show that in the energy range from the Fe $K$-edge to the $\mathrm{Gd} L_{2}$-edge $(7-8 \mathrm{keV})$ the diamond slab transmitted $\sim 30 \%$ of the incident beam, in agreement with the theoretical predictions. ${ }^{2}$

The $\mathrm{Gd} L_{2}$-edge $\left(7930 \mathrm{eV}\right.$ ) XMCD spectra of $\mathrm{GdFe}_{2}$ shown in Fig. 6 were recorded at room temperature and under an applied magnetic field of $4.8 \mathrm{kOe}$. Both magnetic field and photon helicity reversal techniques were used employing an angular offset equal to 90 arcsec. In all of the cases we have adopted the same convention to display the spectra: the XMCD signal corresponds to the spin-dependent absorption coefficient obtained as the difference of the absorption coefficient $\mu_{\mathrm{c}}=\left(\mu^{-}-\mu^{+}\right)$for antiparallel, $\mu^{-}$, and parallel, $\mu^{+}$, orientations of the photon helicity and the magnetic field applied to the sample. A comparison of the results obtained using both methods is reported in Fig. 6, where the XMCD signals have been normalized to the absorption jump and corrected by the estimated circular polarization rate $(\sim 0.8)$. Both measuring methods yield the same spectral shape characterized by a negative peak, $\sim 5 \mathrm{eV}$ wide, centered at $\sim 1 \mathrm{eV}$ above the edge, in agreement with previous results (LagunaMarco et al., 2005, 2008a,b). For further verification of the reliability of these results, the same specimen was measured in transmission mode at the undulator beamline BL39XU at SPring-8 (proposal No. 2008A1051). The helicity modulation technique was used with a $0.7 \mathrm{~mm}$-thick diamond phase retarder. The good agreement between both measurements, reported in Fig. 6, points out the high performance of the SpLine set-up.

Similar results have been obtained in the case of the $\mathrm{Ho}_{2_{2}}$ edge $(8918 \mathrm{eV}) \mathrm{XMCD}$ of $\mathrm{HoFe}_{2}$. The XMCD signal was

\footnotetext{
${ }^{2}$ Theoretical predictions give a transmission of $\sim 20 \%$ and $\sim 35 \%$ at 7 and $8 \mathrm{keV}$, respectively, for a diamond slab in the Laue (220) configuration.
}

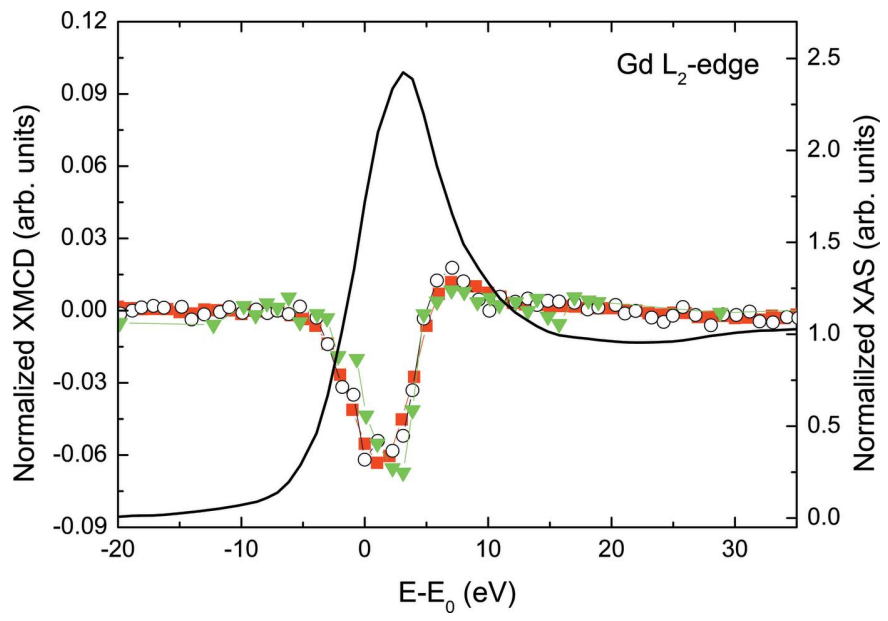

Figure 6

Normalized Gd $L_{2}$-edge XMCD spectra recorded on $\mathrm{GdFe}_{2}$ using field reversal (green solid triangle), helicity reversal (black open circle) and helicity modulation (red solid square) configurations (see text for details). The normalized XANES spectrum is also shown (black line). This figure is in colour in the electronic version of this paper.

recorded by using the helicity reversal method with an offset of 61 arcsec that corresponds to an estimated circular polarization rate higher than 0.7. As shown in Fig. 7, the XMCD signals recorded on the same specimen at both SpLine and BL39XU show a remarkable agreement: the Ho $L_{2}$-edge $\mathrm{XMCD}$ exhibits a positive peak at $\sim 1 \mathrm{eV}$ above the edge, a negative peak at $\sim 4 \mathrm{eV}$ and another positive peak centered at $\sim 7 \mathrm{eV}$ above the edge. The measurements at BL39XU were performed under the same experimental conditions as mentioned for the $\mathrm{Gd} L_{2}$-edge case. It should be noted, however, that the amplitude of the spectrum recorded in our experimental set-up is slightly smaller than that recorded at BL39XU. This effect might be addressed to the loss of the correct XPR position during the measurement since no

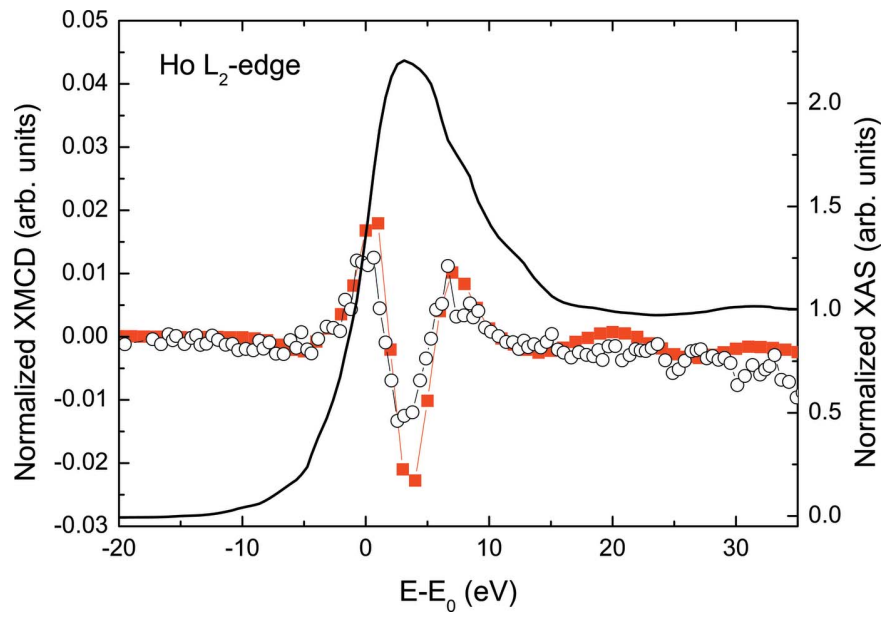

Figure 7

Comparison of normalized Ho $L_{2}$-edge XMCD signals recorded on $\mathrm{HoFe}_{2}$ by using the helicity reversal (black open circle) and helicity modulation techniques (red solid square). The normalized XANES spectrum is also shown (black line). This figure is in colour in the electronic version of this paper. 


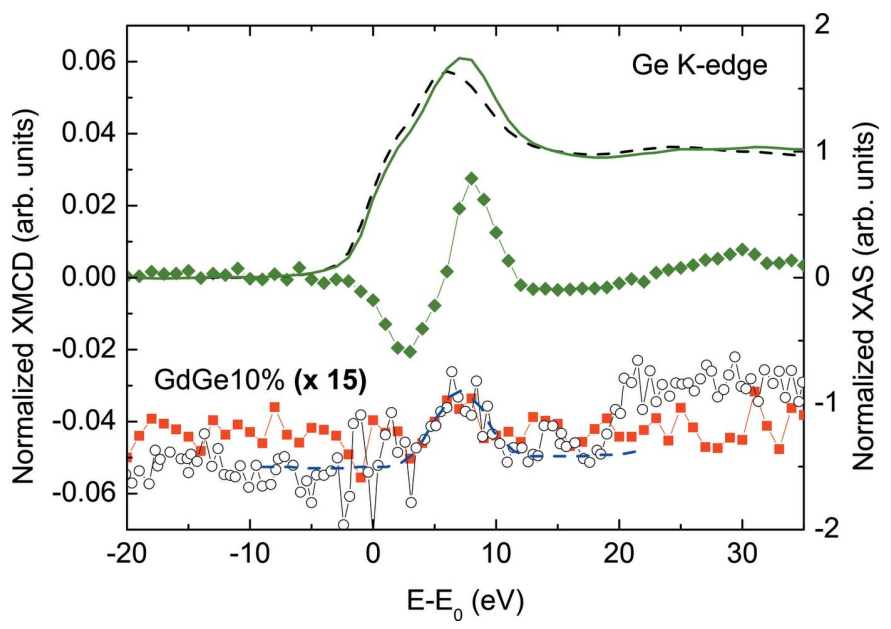

\section{Figure 8}

Comparison of normalized XMCD signals of $\mathrm{Gd}\left(\mathrm{Fe}_{0.9} \mathrm{Ge}_{0.1}\right)_{2}$ recorded at the Ge $K$-edge using helicity reversal (black open circles) and helicity modulation techniques (red solid squares). The dashed (blue) line is a guide to the eye. The XMCD signal of $\mathrm{Ho}\left(\mathrm{Fe}_{0.9} \mathrm{Ge}_{0.1}\right)_{2}$ at $5 \mathrm{~K}$ and $50 \mathrm{kOe}$ is also shown (green solid diamonds). For the sake of completion the normalized XANES spectra is also shown for $\mathrm{Gd}\left(\mathrm{Fe}_{0.9} \mathrm{Ge}_{0.1}\right)_{2}$ (black dashed line) and $\mathrm{Ho}\left(\mathrm{Fe}_{0.9} \mathrm{Ge}_{0.1}\right)_{2}$ (green line). This figure is in colour in the electronic version of this paper.

encoder was used for the rotation. However, this hypothesis can be discarded because the diffraction peaks measured after the XMCD measurement are close to the expected values. Therefore, we tentatively assign this reduction to the different harmonic rejection method used (second crystal of the DCM detuning at SpLine and flat Rh-coated mirror after the XPR at BL39XU) since the high-order harmonics contamination can distort the XMCD measurement.

Finally, in order to test the set-up at high energy, i.e. when the transmission through the diamond is higher and hence the circular polarization is lower, we have recorded the XMCD at the Ge $K$-edge $(11103 \mathrm{eV})$. To this end we have considered the case of the $\mathrm{Gd}\left(\mathrm{Fe}_{0.9} \mathrm{Ge}_{0.1}\right)_{2}$ Laves phase compound in which magnetic $\mathrm{Fe}$ atoms have been substituted by non-magnetic $\mathrm{Ge}$ atoms. Recent works have shown the existence of non-zero XMCD signals at the $K$-edge of atoms like Ge or Ga (Inada $e t$ al., 2005; Haskel et al., 2007; Chaboy et al., 2009). These results illustrate the importance of XMCD in determining the exact nature of the induced magnetic moments in traditionally nonmagnetic atoms owing to the interplay of the hybridization and of the modification of the electronic structure.

We have recorded the Ge $K$-edge XMCD by using an angular offset of $34 \mathrm{arcsec}$ for which the theoretical estimate yields a circular polarization rate of $\sim 0.6{ }^{3}$ The results are reported in Fig. 8 where the XMCD signal measured in the setup developed at SpLine is compared with that recorded at the XMCD-dedicated station of BL39XU at SPring-8. At

\footnotetext{
${ }^{3}$ We are conscious that the circular polarization rate obtained with a 111diamond of thickness $0.5 \mathrm{~mm}$ in the Laue(220) symmetric geometry is not optimum at the Ge $K$-edge energies, it being more convenient to use a thicker diamond phase plate to obtain a higher degree of circular polarization. However, it serves our purpose of checking the circular polarization rate achieved at higher energy in our experimental set-up.
}

BL39XU the helicity modulation technique with a $1.4 \mathrm{~mm}$ thick diamond XPR was used to record the XMCD signal in transmission mode (proposal No. 2008B1753). In both cases the XMCD spectra show a spectral feature of positive sign centered at $\sim 7 \mathrm{eV}$ above the edge, whose amplitude is only about $0.1 \%$ of the absorption jump. ${ }^{4}$ For the sake of comparison we also show, in Fig. 8, the XMCD signal of $\mathrm{Ho}\left(\mathrm{Fe}_{0.9} \mathrm{Ge}_{0.1}\right)_{2}$ at the $\mathrm{Ge} K$-edge recorded at $5 \mathrm{~K}$ and under a magnetic field of $50 \mathrm{kOe}$. In this case, for the Ho Laves phase compound, the Ge magnetic polarization is larger and the XMCD signal is clearer than in the Gd compound case. We have measured the $\mathrm{Gd}$ compound instead of the Ho compound because the goal was to illustrate the performance of the XMCD set-up at SpLine at the detection limit observed at BL39XU. These results constitute the first evidence of the magnetic polarization of the $\mathrm{Ge}$ atoms in the diluted $R\left(\mathrm{Fe}_{1-x} \mathrm{Ge}_{x}\right)_{2}$ Laves phase materials.

\section{Summary and conclusions}

We have reported the first XMCD measurements performed on the bending-magnet BM25 A-SpLine beamline at the ESRF. Linearly polarized X-rays were converted into circularly polarized X-rays by using a $0.5 \mathrm{~mm}$-thick diamond X-ray phase retarder working in the Laue geometry, and the results are in good agreement with the prediction for the polarization rate. Both magnetic field reversal and helicity reversal modes have been used to record XMCD in an energy range from 7 to $11 \mathrm{keV}$. The quality of the dichroic signals obtained is comparable with that recorded at the XMCD-dedicated beamline BL39XU at SPring-8.

This work was partially supported by a Spanish CICYTMAT2008-06542-C04 grant. MALM and RB acknowledge the Ministerio de Ciencia e Innovación of Spain for their Postdoctoral and $\mathrm{PhD}$ grants, respectively. We also acknowledge the Spanish Ministerio de Ciencia e Innovación and Consejo Superior de Investigaciones Científicas for financial support and for providing synchrotron radiation facilities access, and we would like to thank SpLine staff and BL39XU staff for their assistance during the proposals 25-01-643 and 25-01-678, and 2008A1051 and 2008B1753, respectively. We are indebted to M. Suzuki and N. Kawamura for many enlightening discussions.

\section{References}

Batterman, B. \& Cole, H. (1964). Rev. Mod. Phys. 36, 681-717. Bouchenoire, L., Brown, S. D., Thompson, P., Duffy, J. A., Taylor, J. W. \& Cooper, M. J. (2003). J. Synchrotron Rad. 10, 172-176.

Castro, G. R. (1998). J. Synchrotron Rad. 5, 657-660.

Chaboy, J., Lázpita, P., Barandiaran, J. M., Gutiérrez, J., FernándezGubieda, M. L. \& Kawamura, N. (2009). J. Phys. Condens. Matter, 21, 016002 .

\footnotetext{
${ }^{4}$ The Ge $K$-edge signal recorded at SpLine shows a poorer signal-to-noise ratio than that recorded at BL39XU where a $1.4 \mathrm{~mm}$-thick XPR, providing a higher circular polarization rate than the $0.5 \mathrm{~mm}$-thick XPR of SpLine, was used.
} 
Duke, P. J. (2000). Synchrotron Radiation: Production and Properties. Oxford Science Publications.

Giles, C., Malgrange, C., Goulon, J., de Bergevin, F., Vettier, C., Dartyge, E., Fontaine, A., Giorgetti, C. \& Pizzini, S. (1994). J. Appl. Cryst. 27, 232-240.

Goulon, J., Brookes, N. B., Gauthier, C., Goedkoop, J. B., GoulonGinet, C., Hagelstein, M. \& Rogalev, A. (1995). Physica B, 208-209, 199-202.

Haskel, D., Lee, Y. B., Harmon, B. N., Islam, Z., Lang, J. C., Srajer, G., Mudryk, Y. K. A. Gschneidner, J. \& Pecharsky, V. K. (2007). Phys. Rev. Lett. 98, 247205.

Haskel, D., Srajer, G., Lang, J. C., Pollmann, J., Nelson, C. S., Jiang, J. S. \& Bader, S. D. (2001). Phys. Rev. Lett. 87, 207201.

Hirano, K., Ishikawa, T., Koreeda, S., Fuchigami, K., Kanzai, K. \& Kikuta, S. (1992). Jpn. J. Appl. Phys. 31, L1209-L1211.

Hirano, K., Izumi, K., Ishikawa, T., Annaka, S. \& Kikuta, S. (1991). Jpn. J. Appl. Phys. 30, L407-L410.

Hirano, K., Izumi, K., Ishikawa, T., Annaka, S. \& Kikuta, S. (1994). Jpn. J. Appl. Phys. 33, L689-L692.

Inada, Y., Honma, T., Kawamura, N., Suzuki, M., Miyagawa, H., Yamamoto, E., Haga, Y., Okane, T., Fujimori, S. \& Onuki, Y. (2005). Physica B, 359-361, 1054-1056.

Ishikawa, T. (1988). Rev. Sci. Instrum. 60, 2058-2061.

Kawata, H., Miyahara, T., Yamamoto, S., Shioya, T., Kitamura, H., Sato, S., Asaoka, S., Kanaya, N., Iida, A., Mikuni, A., Sato, M.,
Iwazumi, T., Kitajima, Y. \& Ando, M. (1988). Rev. Sci. Instrum. 60, $1885-1888$.

Laguna-Marco, M. A., Chaboy, J. \& Piquer, C. (2008a). Phys. Rev. B, 77, 125132.

Laguna-Marco, M. A., Chaboy, J. \& Piquer, C. (2008b). J. Appl. Phys. 103, 07E141.

Laguna-Marco, M. A., Chaboy, J., Piquer, C., Maruyama, H., Ishimatsu, N., Kawamura, N., Takagaki, M. \& Suzuki, M. (2005). Phys. Rev. B, 72, 052412.

Lang, J. C. \& Srajer, G. (1995). Rev. Sci. Instrum. 66, 1540-1542.

Maruyama, H. (2001). J. Synchrotron Rad. 8, 125-128.

Pizzini, S., Bonfim, M., Baudelet, F., Tolentino, H., San Miguel, A., Mackay, K., Malgrange, C., Hagelstein, M. \& Fontaine, A. (1998). J. Synchrotron Rad. 5, 1298-1303.

Schütz, G., Wagner, W., Wilhelm, W., Kienle, P., Zeller, R., Frahm, R. \& Materlik, G. (1987). Phys. Rev. Lett. 58, 737-740.

Stöhr, J., Padmore, H. A., Anders, S., Stammler, T. \& Scheinfein, M. R. (1998). Surf. Rev. Lett. 5, 1297-1308.

Suzuki, M., Kawamura, N. \& Ishikawa, T. (2003). Rev. Sci. Intrum. 74, L1488-L1490.

Suzuki, M., Kawamura, N., Mizumaki, M., Urata, A., Maruyama, H., Goto, S. \& Ishikawa, T. (1998). Jpn. J. Appl. Phys. 37, L1488L1490.

Yamamoto, S., Kawata, H., Kitamura, H., Ando, M., Saki, N. \& Shiotani, N. (1989). Phys. Rev. Lett. 62, 2672. 